\title{
The multifractal structure of satellite sea surface temperature maps can be used to obtain global maps of streamlines
}

\author{
A. Turiel ${ }^{1}$, V. Nieves ${ }^{1}$, E. Garcia-Ladona ${ }^{1}$, J. Font ${ }^{1}$, M.-H. Rio ${ }^{2}$, and G. Larnicol ${ }^{2}$ \\ ${ }^{1}$ Institut de Ciències del Mar, CSIC, Barcelona, Spain \\ ${ }^{2}$ CLS - Space Oceanography Division, Toulouse, France
}

Received: 18 November 2008 - Published in Ocean Sci. Discuss.: 22 January 2009

Revised: 1 September 2009 - Accepted: 5 October 2009 - Published: 26 October 2009

\begin{abstract}
Nowadays Earth observation satellites provide information about many relevant variables of the ocean-climate system, such as temperature, moisture, aerosols, etc. However, to retrieve the velocity field, which is the most relevant dynamical variable, is still a technological challenge, specially in the case of oceans. New processing techniques, emerged from the theory of turbulent flows, have come to assist us in this task. In this paper, we show that multifractal techniques applied to new Sea Surface Temperature satellite products opens the way to build maps of ocean currents with unprecedented accuracy. With the application of singularity analysis, we show that global ocean circulation patterns can be retrieved in a daily basis. We compare these results with high-quality altimetry-derived geostrophic velocities, finding a quite good correspondence of the observed patterns both qualitatively and quantitatively; and this is done for the first time on a global basis, even for less active areas. The implications of this findings from the perspective both of theory and of operational applications are discussed.
\end{abstract}

\section{Introduction}

Earth observation satellites provide an excellent platform for continuously monitoring the climatic evolution of our planet. Present remote sensors provide, on a routinary basis and at global scales, a wide set of measured variables such as Sea Surface Temperature (SST), water vapor content in atmosphere, ocean surface chlorophyll concentration, aerosol concentration in air and a long etc. Atmospheric and ocean studies have largely been benefited from it, although the characterization of ocean dynamics by means of satellite observations is however more elusive than that of atmosphere. First,

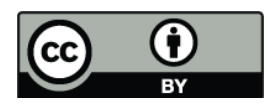

Correspondence to: A. Turiel (turiel@cmima.csic.es) because due to the highest optical extinction of ocean water, our satellite-based knowledge about the ocean is limited to a narrow layer close to surface, of a depth going from millimeters to a few meters. Second, because despite some recent developments in Doppler radar shift (Chapron et al., 2005; Johannessen et al., 2005), to directly obtain a crucial dynamic variable as the ocean velocity field from satellites is still a challenging task.

Velocities can be retrieved through the Sea Surface Height (SSH) measurements from radar altimetry. The SSH field is linked to the pressure field and then the geostrophic approximation may be used to derive the velocity field. As a result quasi-synoptic maps can be build through the interpolation of several altimeters (LeTraon et al., 1998) and have been used to study the ocean variability at relatively large scales (Wunsch and Stammer, 1998). Sampling limitations as well as the necessity to combine the signals of several altimeters limit the spatial and time resolutions and prevent altimetry maps to resolve part of the relevant oceanic processes (Pascual et al., 2006).

An alternative strategy to evaluate ocean surface velocities from satellite data is to process sequences of images of SST (Bowen et al., 2002) or other scalars (Crocker et al., 2007). These techniques are based on tracking ocean structures which have been generated by the flow and are still being dragged (advected) by it. This strategy leads to useful velocity fields, although the spatial and temporal resolutions are relatively limited due to processing needs, and sometimes the field is not well resolved. However, satellite images of scalar variables can still be further exploited to gain insight about the dynamics, taking advantage of the turbulent structure of ocean flows.

When turbulence develops in a flow, a very complicated structure raises. In a turbulent flow, intermittency is revealed as dramatic changes of velocity and other properties as one moves across the fluid domain. As a consequence, shear is dominant over many areas; scalar parcels dragged

Published by Copernicus Publications on behalf of the European Geosciences Union. 
by two different filaments rapidly separate from each other and so the flow is continuously creating new singularity fronts. By singularity we understand that the value of the local singularity exponent (a measure of the function regularity (Daubechies, 1992; Turiel and Parga, 2000)) decreases, what means that the function becomes more irregular. Therefore, in a scalar submitted to turbulence singularities are created in a statistically steady rate. Hence, each time a singularity is observed in a scalar it would probably indicate the presence of a strong velocity gradient (Kraichnan, 1968; Lapeyre et al., 2001). In fact, in previous works (Turiel et al., 2005b; Isern-Fontanet et al., 2007; Turiel et al., 2008a) some authors have argued that extracting singularities from satellite images as SST maps serves to delineate flow streamlines. Expressed in other words, singularity exponents are created by the flow along streamlines, what is an appropriate assumption as far as the stirring by the horizontal advection is the main singularity-inducing effect. This hypothesis is supported by the facts that at the mesoscale ocean flows are practically bi-dimensional and dominated by geostrophic balance and both SST and Chlorophyll images exhibit a common turbulent signature (Nieves et al., 2007).

In this paper, we will show for the first time that singularity exponents derived from microwave SST maps serve to trace streamlines of surface currents in any situation, not only for areas with intense mesoscale activity as done in previous works (Turiel et al., 2005b, 2008a; Isern-Fontanet et al., 2007), but for the whole global ocean and any situation. This result demonstrates that singularity advection is a robust, general principle, at least for scales around and larger than mesoscale. A key point in this verification is the use of a new generation of altimeter products, in which a sufficient number of altimeters are combined together to generate maps with good both time and spatial resolutions. Selfconsistently, this work serves to validate the quality of these altimeter products. Additionally, we precisely quantify the accuracy of the obtained streamlines by estimating the rate at which they diverge from the actual streamlines.

The paper is structured as follows. In Sect. 2 we will present the data to be used in this study. Then, in Sect. 3 the concept of singularity exponent field of a scalar map is introduced and discussed, and some examples are shown. We thus proceed to Sect. 4, where the streamlines derived from singularity analysis of SST maps are compared with altimetry-derived geostrophic currents. Finally, the conclusions are presented in Sect. 5.

\section{Description of the data}

Our main source of data for this study are Optimally Interpolated (OI) SST images from Microwave (MW) Radiometer SSTs. Microwave OI SST data are produced by Remote Sensing Systems and sponsored by National Oceanographic Partnership Program (NOPP), the NASA Earth Sci- ence Physical Oceanography Program, and the NASA REASoN DISCOVER Project. Data are available through the following web site: http://www.remss.com.

As SST images contain irregularly spaced data (in time and space) due to orbital gaps or environmental conditions, an interpolation of the data onto a regularly sampled grid is needed to make up for this missing data. MW SST products accurately resolve some features that could be missed due to data gaps or weather condition. This is possible by blending TMI and AMSR-E SSTs, providing nearly complete global coverage each day. Near real time OI SST products are created daily, even if no new observations exist. However, the product is $0.25 \times 0.25$ degree gridded, which is a coarse resolution in comparison with the standard infrared SSTs one. Processing details can be found in (Reynolds and Smith, 1994) and at the following website: http://www.ssmi.com/sst/microwave_oi_sst_browse.html.

The second source of data for this study are geostrophic surface currents computed at CLS in the framework of the SURCOUF project (Larnicol et al., 2006). Two types of currents maps are produced by SURCOUF. First, real time global maps of surface currents, which are produced daily on a $1 / 3^{\circ}$ Mercator grid. Second, a reanalysis of these currents exists for the period June 1999-January 2006. In this study, the SURCOUF daily delayed-time maps of absolute geostrophic surface currents are used for the period September 2002 to August 2003. This period is particularly interesting since four altimetric satellites (Jason-1, ERS2/ENVISAT, TOPEX interleaved, GFO) were working together, allowing a much improved description of the ocean mesoscale (Pascual et al., 2006). In fact, only with the use of SURCOUF maps we can have an accurate estimation of surface currents at a daily basis instead of the usual 10-day time span. This point is critical for our study because, as shown in IsernFontanet et al. (2007), singularity analysis of SST maps reveals that for many areas changes at mesoscale are noticeable after just three days.

SURCOUF currents are based on the use of the altimetric data distributed by AVISO (http://www.aviso.oceanobs.com) and processed through the following steps: First, the usual geophysical corrections were applied to the altimetric heights from the four satellites (apart from GFO, for which specific corrections were applied (LeTraon et al., 2003)) and Sea Level Anomalies were computed subtracting from the instantaneous altimetric heights a 7 year (1993-1999) mean profile. Specific processing was applied to TP interleaved and GFO to achieve consistency with the Jason-1 and ERS2-ENVISAT missions (LeTraon et al., 2003; LeTraon and Dibarboure, 2004). Then the along-track anomalies from the four different missions were mapped into a global $1 / 3^{\circ}$ resolution Mercator grid using a procedure described in (LeTraon et al., 2003) and the observed Combined Mean Dynamic Topography RIO05 (Rio et al., 2005) was added to the SLA maps in order to retrieve daily maps of absolute ocean topography. The surface velocity currents were finally computed from the 
absolute topography maps using the geostrophic assumption. In the equatorial band the quasi geostrophic approximation is applied (Lagerloef et al., 1999).

\section{Characterization of streamlines by singularity exponents}

The first step in our work is to design stable, highperformance tools to perform singularity analysis on real images, capable to assign an accurate value of singularity exponent at each point. The singularity exponent of a scalar at a given point is a scale-invariant, dimensionless measure of the degree of regularity or irregularity of the function at that point (see Isern-Fontanet et al., 2007, and Turiel et al., 2008a, for a full discussion of the concept). As furnished by the acquisition devices, images (properly speaking, 2-D maps of a given variable) do not vary continuously on space but are sampled on a discrete grid, and are also affected by several sources of error, noise and acquisition problems. Hence, singularity analysis must implement appropriate filtering and interpolation schemes (Daubechies, 1992; Arneodo et al., 1995).

In this paper, we have used the same strategy developed in (Turiel and Parga, 2000), which has been shown to attain good spatial and value accuracy in the determination of singularity exponents in many contexts and in particular for the processing of satellite imagery (Turiel et al., 2005b,a; IsernFontanet et al., 2007; Nieves et al., 2007; Turiel et al., 2008a). We will denote the scalar under study by $\theta(\mathbf{x})$ (where $\theta$ can be SST, chlorophyll concentration, etc., and $\mathbf{x}$ denotes the point in the image plane). At each location $\mathbf{x}$ the singularity exponent $h(\mathbf{x})$ can be obtained by processing the wavelet projections (Daubechies, 1992; Mallat, 1999) of the modulus of the gradient of $\theta$, that we denote by $T_{\Psi}[|\nabla \theta|](\mathbf{x}, r)$ and are defined as follows:

$$
T_{\Psi}[|\nabla \theta|](\mathbf{x}, r) \equiv \int d \mathbf{x}^{\prime}|\nabla \theta|\left(\mathbf{x}^{\prime}\right) \frac{1}{r^{2}} \Psi\left(\frac{\mathbf{x}-\mathbf{x}^{\prime}}{r}\right)
$$

As shown in previous works (Turiel et al., 2005b; IsernFontanet et al., 2007; Nieves et al., 2007) the wavelet projection of gradients of SST and other scalars depend on the scale resolution parameter $r$ as a power-law, characterized by the local singularity exponent $h(\mathbf{x})$ in the way:

$$
T_{\Psi}[|\nabla \theta|](\mathbf{x}, r)=\alpha(\mathbf{x}) r^{h(\mathbf{x})}+o\left(r^{h(\mathbf{x})}\right)
$$

where the expression $o\left(r^{h(\mathbf{x})}\right)$ means a term which is negligible compared to $r^{h(\mathbf{x})}$ when $r^{h(\mathbf{x})}$ goes to zero. Scalars submitted to turbulence present local power-law scaling at each one of its points as the one expressed by Eq. (2). This is connected to the Microcanonical Multifractal Formalism (Turiel et al., 2008b): Eq. (2) implies that $\theta$ is multifractal (i.e., a composite of multiple fractal interfaces) and at the same time allows to explicitly separate each fractal interface from a given signal $\theta(\mathbf{x})$ (in contrast with classical approaches, which only allow a statistical characterization of the fractal components (Frisch, 1995)).

For the determination of the singularity exponents we have employed as wavelet $\Psi$ an optimized numerical implementation of the Lagrangian wavelet,

$\Psi_{L}(\mathbf{x})=\frac{1}{1+|\mathbf{x}|^{2}}$

Such a function is not an admissible wavelet (Daubechies, 1992) because it is strictly positive and hence it cannot be used to represent data. However, as discussed in Turiel et al. (2008b), positive wavelets can be used to obtain the singularity exponents of multifractal measures as the ones defined by gradient modulus, so $\Psi_{L}$ can be used to this purpose. In fact, it has been shown to have a good performance on real situations, although it truncates the singularities beyond $h=0$. To avoid this effect, which is connected with the behavior of the tail of the wavelet (Turiel et al., 2008b), we have constructed a numerical implementation, $\Psi_{L n}$, which is defined by a matrix of numerical weights which is close to $\Psi_{L}$ for small values of $|\mathbf{x}|$ but has a faster decay for larger values of $|\mathbf{x}|$.

The exponents $h(\mathbf{x}, t)$ are obtained by the application of Eq. (2) at different resolution scales $r$. For a set of scales $r_{1}, r_{2}, \ldots, r_{m}$ a linear regression of $\log T_{\Psi}[\nabla \theta](\mathbf{x}, r)$ vs. $\log r$ is performed at each point $\mathbf{x}$ in the image; the slope of such a regression is the singularity exponent $h(\mathbf{x})$. For the experiences shown in this paper we have used $m=7$ different scales which are uniformly sampled in a logarithmic axis, $\log r_{i+1}-\log r_{i}=$ constant. We fix the constant so that $r_{1}=1$ pixel and $r_{m}=0.1 \times$ image size.

In Fig. 1 we show an example of singularity analysis on a global map of Microwave (MW) Sea Surface Temperature (SST). Many hydrographic features of global and regional ocean circulation become evident in the singularity map. Main boundary currents, such as the Gulf Stream, the Kuroshio, the Agulhas retroflection current or the Falkland current, as well as the diverse filaments of the Antartic Circumpolar Current, which can be vaguely distinguished in SST maps, become clear and distinct in the singularity map, in addition with other emerging filaments, eddies and currents that were hidden in the SST maps. One of the striking properties of singularity analysis is that it is able to track in a stable way a streamline, even if the amplitude of the transition is rather small and the signal-to-noise ratio of the data rather low. The reason for this is that singularity exponents characterize the sharpness of a transition, disregarding the amplitude of the variation, because they are dimensionless quantities. Besides, transitions can be observed even in the presence of relatively large spatially uncorrelated noise if they are spatially coherent, because according to Eq. (2) singularity exponents are obtained after processing a region of size $r$ (and not only a point). Notice that even accepting 

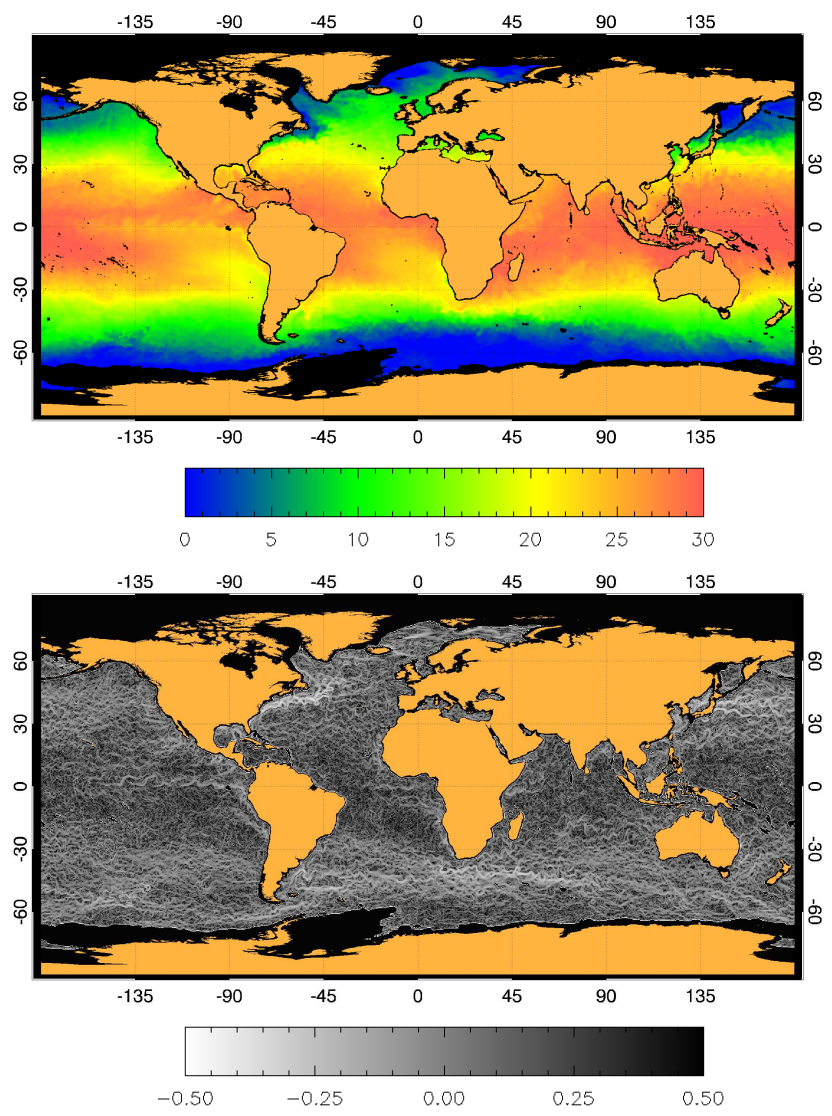

Fig. 1. Top: Global MW SST image for 1 January 2005; Bottom: Associated map of singularity exponents.

that singularity exponents serve to delineate the streamlines of the flow, they do not offer information about the velocity modulus or sense, only about its direction. However, this information is already very useful to understand ocean circulation. In the following we will discuss on the validation of singularity streamlines.

\section{Comparison with altimetry}

\subsection{Qualitative comparison}

Although the singularity maps derived from MW SST that we have presented are appealing and seem to be highly correlated with the geometrical arrangement of currents in oceans, we need to confirm their validity as current tracers. Hence, we need independent measurements to contrast the similitudes and to quantify the degree of closeness between ocean currents and the filaments shown in singularity maps. However, this is precisely the question: we do not have synoptic maps of ocean currents apart from altimetry maps, and these are poorly resolved in time. Nevertheless, for more than a year between 2002 and 2003 high-quality daily maps of geostrophic currents derived from the combination of four satellite altimeters are available (Pascual et al., 2006). We use these data (SURCOUF maps) in our study.

In Fig. 2 we show a couple of examples of the comparison of singularity maps derived from MW SST and SURCOUF altimeter maps, for two different regions. The visual assessment indicates that singularities align quite well with altimeter-derived geostrophic currents. We present excerpts of the same two cases for two smaller subregions with less active currents in Fig. 3. Although over these two regions the acquisitions have smaller Signal-to-Noise Ratios (in the case of altimeter because the smaller changes in dynamic height, and in the case of SST because these regions have gradients of smaller amplitude) and interpolation tends to smooth away details, correspondence is still rather good. Notice that nevertheless all the effects mentioned above should be taken into account when designing a quantitative measure of the degree of closeness.

\subsection{Evaluation of time derivatives}

A simple visual comparison does not allow to quantify the degree of closeness between altimeter-derived currents and singularity lines. More quantitative criteria can be devised by the evaluation of time derivatives of the field of singularity exponents, and its capability of resolving streamlines can be compared with respect to other tracers, as SST itself. However, SST and singularity exponents have a sampling grid which is different from that of altimeter-derived velocities, which in addition has an angular spacing which varies with latitude. A simple linear interpolation of the scalars to the velocity grid will considerably damage the spatial coherency of the very intermittent patterns derived by singularity analysis, because singularity analysis is a non-linear method. For that reason, a careful design of the numerical strategy to estimate time derivatives is required, as we explain in the following.

For the determination of the advective and material time derivatives of a given scalar we need to compute Lagrangian trajectories, for which we have used a simple integration scheme. Let us first introduce some notation. We will denote the longitude coordinate by $\phi$ and the latitude coordinate by $\lambda$. For two points on the sphere $\mathbf{p}=(\phi, \lambda), \mathbf{p}^{\prime}=\left(\phi^{\prime}, \lambda^{\prime}\right)$ we define the distance between them by the length of arc of geodesic circle which joints both points. For two points of close coordinates we approximate this distance $d\left(\mathbf{p}, \mathbf{p}^{\prime}\right)$ by the following expression:

$d\left(\mathbf{p}, \mathbf{p}^{\prime}\right)=R_{e} \sqrt{\left(\lambda-\lambda^{\prime}\right)^{2}+\left(\phi-\phi^{\prime}\right)^{2} \cos ^{2}\left(\frac{\lambda+\lambda^{\prime}}{2}\right)}$

where $R_{e}$ is the radius of Earth and the angular variables are expressed in radians.

Given a point $\mathbf{p}$ in the sphere, we evaluate the velocity at that point by interpolating the velocities of the four closest points. If the four first neighbors of $\mathbf{p}$ on the velocity grid are 

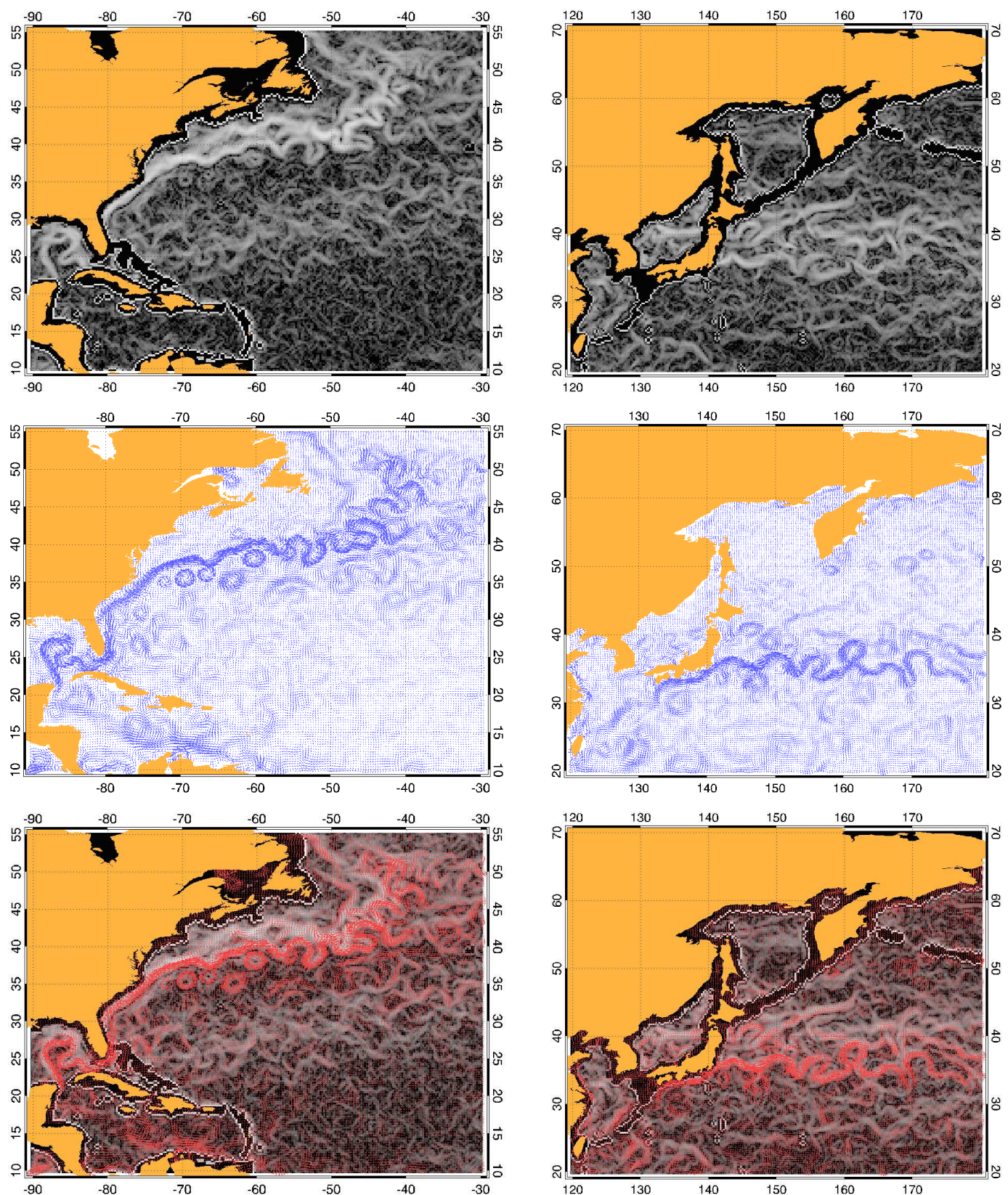

Fig. 2. From top to bottom: Singularity exponents derived from MW SST for two different areas and times; altimeter-derived geostrophic velocities for the same locations and times; and superimposition of both fields. The colorbar for the singularity exponent maps is the same as in Fig. 1; the maximum length of velocity vectors corresponds to $1 \mathrm{~m} / \mathrm{s}$. Results on the left column are for Gulf Stream area on 1 February 2003; results on the right are for Kuroshio current on 1 November 2002.

the points $\mathbf{q}_{1}, \mathbf{q}_{2}, \mathbf{q}_{3}$ and $\mathbf{q}_{4}$, we evaluate the velocity at $\mathbf{p}$ as follows:

$\mathbf{v}(\mathbf{p}, t)=\sum_{i \text { valid }} \frac{Z}{d\left(\mathbf{p}, \mathbf{q}_{i}\right)} \mathbf{v}\left(\mathbf{q}_{i}, t\right)$

where the sum in the expression above is restricted to valid points (i.e., points on the ocean with measured velocity) and the normalization constant $Z$ is such that all the weights sum up to 1 ,

$$
Z^{-1}=\sum_{i \text { valid }} \frac{1}{d\left(\mathbf{p}, \mathbf{q}_{i}\right)}
$$

When none of the four first neighbor points has a valid velocity we consider the point $\mathbf{p}$ cannot be assigned a valid velocity. 

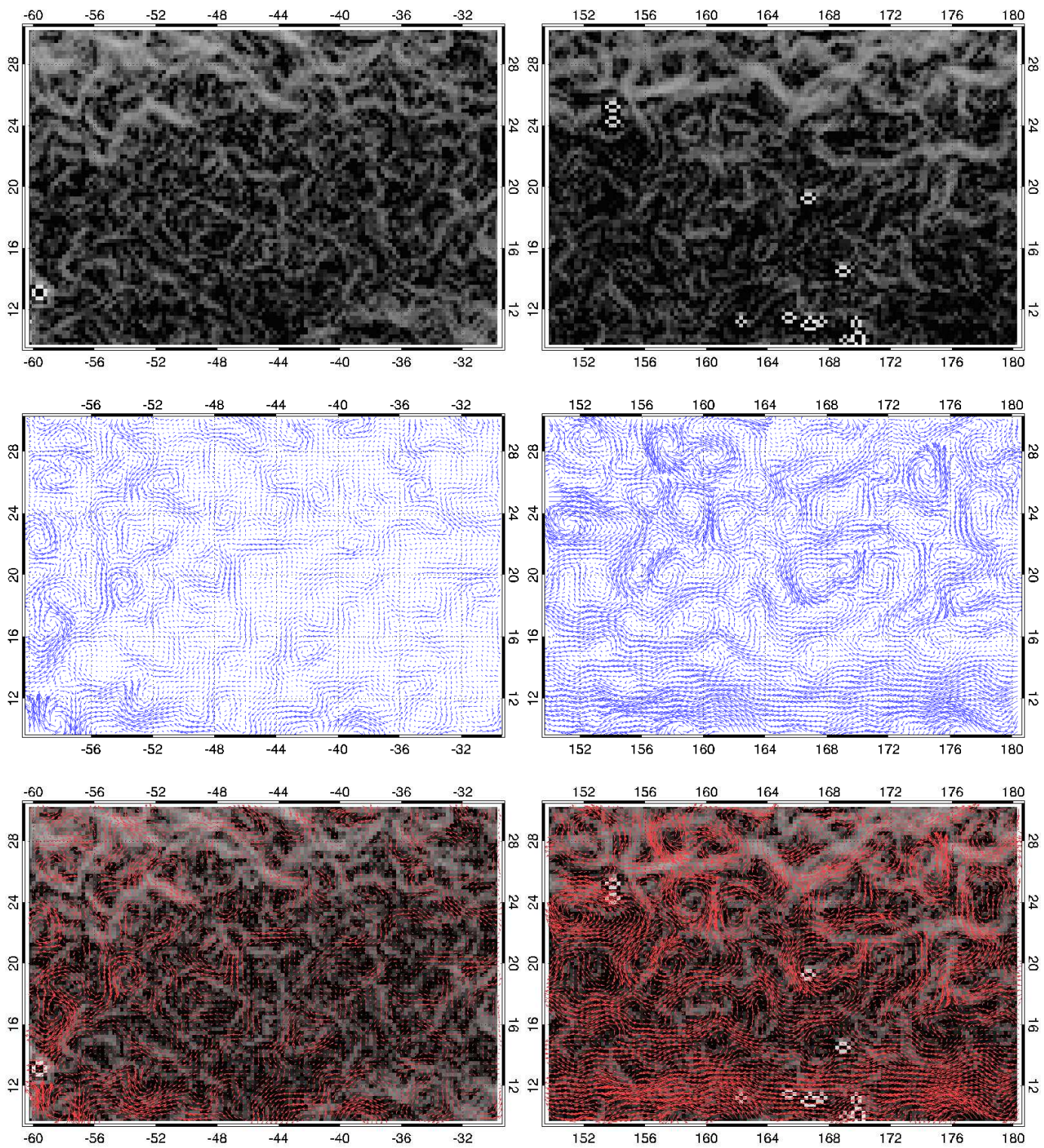

Fig. 3. Excerpts from Fig. 2 over the less active areas.

A trajectory $\mathbf{p}(t)$ is constructed by integrating velocity maps, interpolated in both space and time, with one-hour time increments, that is:

$\mathbf{p}(t+\Delta t)=\mathbf{p}(t)+\mathbf{v}(\mathbf{p}(t), t) \Delta t$

where here $\Delta t=1 \mathrm{~h}$.

To compute the advective and material derivatives of a scalar $\theta(\mathbf{x}, t)$ we need to compute its increments along a trajectory for constant and time-varying maps, respectively. We evaluate the value of the scalar $\theta$ at a non-grid point $\mathbf{p}$ in a similar way to what is done with the velocity, namely:

$\theta(\mathbf{p}, t)=\sum_{i \text { valid }} \frac{Z}{d\left(\mathbf{p}, \mathbf{q}_{i}\right)} \theta\left(\mathbf{q}_{i}, t\right)$
The interpolation on singularity exponents should be treated in a slightly different way, however. When considering singularity exponents $h(\mathbf{x}, t)$ it should be taken into account that variables $h(\mathbf{x}, t)$ are not additive and hence they cannot be linearly interpolated. According to Eq. (2), what is additive is $r^{h(\mathbf{x}, t)}$, so we should hence interpolate $h(\mathbf{p}, t)$ according to the following expression:

$r^{h(\mathbf{p}, t)}=\sum_{i \text { valid }} \frac{Z}{d\left(\mathbf{p}, \mathbf{q}_{i}\right)} r^{h\left(\mathbf{q}_{i}, t\right)}$

where $r$ is the resolution scale at which singularity exponents are calculated. The value of $r$ can be difficult to obtain in real situations, but we can take advantage of the fact it is very 

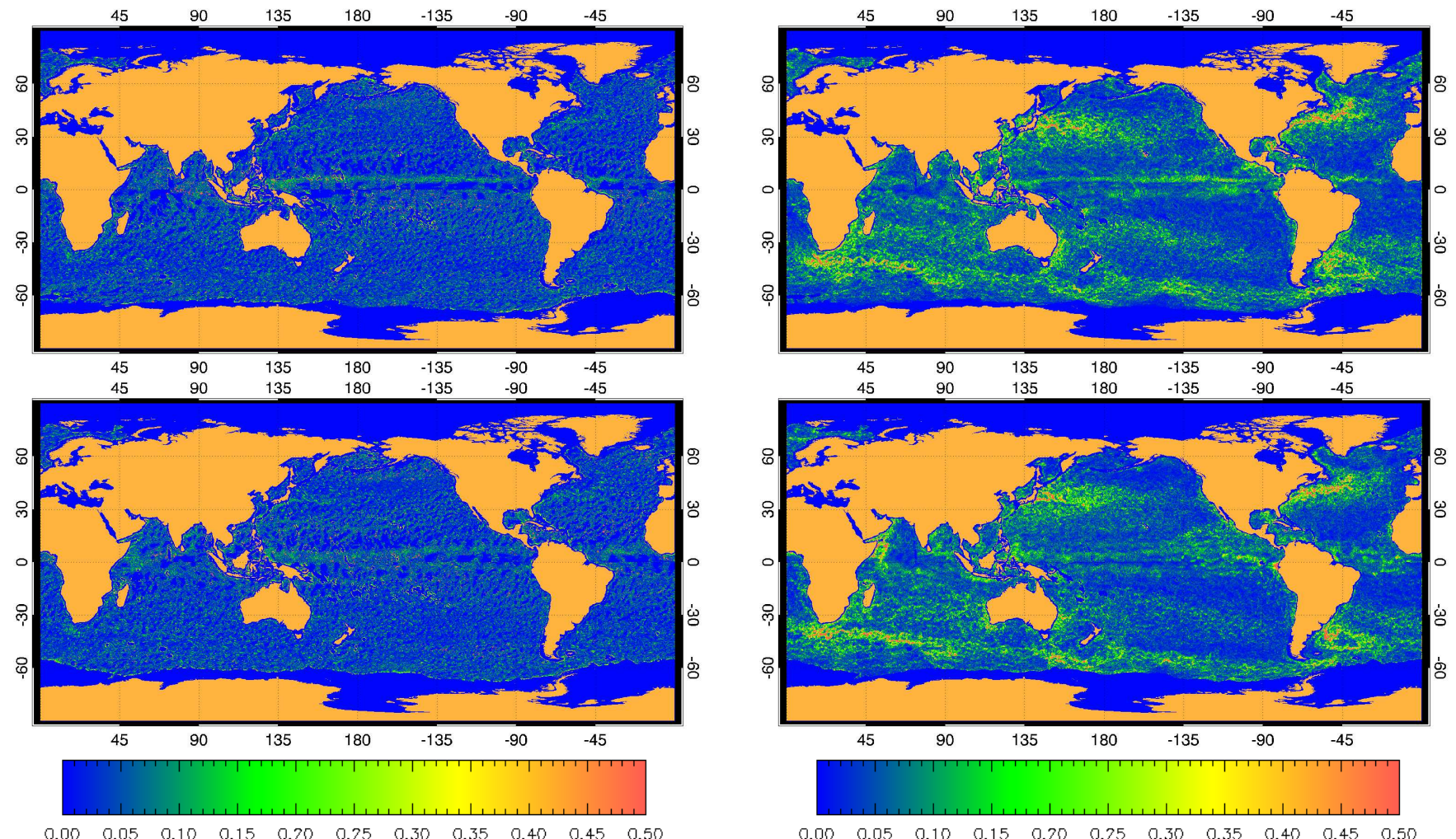

day $^{-1}$

${ }^{o} \mathrm{C} / \mathrm{day}$

Fig. 4. Time averages of the absolute value of advective derivatives of singularity exponent maps (left) and SST (right), for a period of three consecutive days. Results on the top row correspond to the period 1-3 January 2003; results on the bottom row are for 1-3 July 2003. The spatial averages of these quantities are as follows: Singularity exponents: $0.065 \mathrm{day}^{-1}$ (January) and $0.068 \mathrm{day}^{-1}$ (July); SST: $0.53^{\circ} \mathrm{C} / \mathrm{day}^{-1}$ (January) and $0.51^{\circ} \mathrm{C} /$ day (July).

small in our case, so we can simplify the expression above by considering the dominant term,

$h(\mathbf{p}, t)=\min _{i \text { valid }}\left\{h\left(\mathbf{q}_{i}, t\right)\right\}$

that is, the exponent at the point $\mathbf{p}$ is the minimum of the exponents of the valid neighboring points.

The advective derivative of $\theta$ at the point $\mathbf{p}(t)$ and time $t$ is given by the variation of $\theta$ along the trajectory for a constant map and time increment $\Delta t=1$ day, according the equation:

$A \theta(\mathbf{p}(t), t)=\frac{\theta(\mathbf{p}(t+\Delta t), t)-\theta(\mathbf{p}(t), t)}{\Delta t}$

while the material derivative is evaluated taking into account that the map $\theta$ itself evolves,

$D \theta(\mathbf{p}(t), t)=\frac{\theta(\mathbf{p}(t+\Delta t), t+\Delta t)-\theta(\mathbf{p}(t), t)}{\Delta t}$

In Figs. 4 and 5, we show the time averages of the absolute values of these derivatives, denoted by $\bar{A} \theta(\mathbf{x}, t)$ and
$\bar{D} \theta(\mathbf{x}, t)$. In the case of the time-averaged advective derivative, at each time $t$ we take each point in the ocean as the origin and we integrate for a single time step $\Delta t$; the advective derivatives at the same point and different times are averaged together. In the case of the time-averaged material derivative, we take each point on the ocean grid as starting point of the respective trajectory, that we follow for all the days in the time period used to average, then the material derivatives starting from the same point at the initial day are averaged together.

\subsection{Comparison of different scalars: divergence speeds}

If the singularity exponents delineate streamlines, then the advective derivative should be zero, $A h=0$. If the singularity exponents are passive tracers, then the material derivative equals zero, $D h=0$. In Figs. 4 and 5, we show examples of the computation of both types of derivative in two different months of the year 2003; notice that in the figures we show the time average for the considered period of the absolute values of the time derivative. For comparison purposes, 

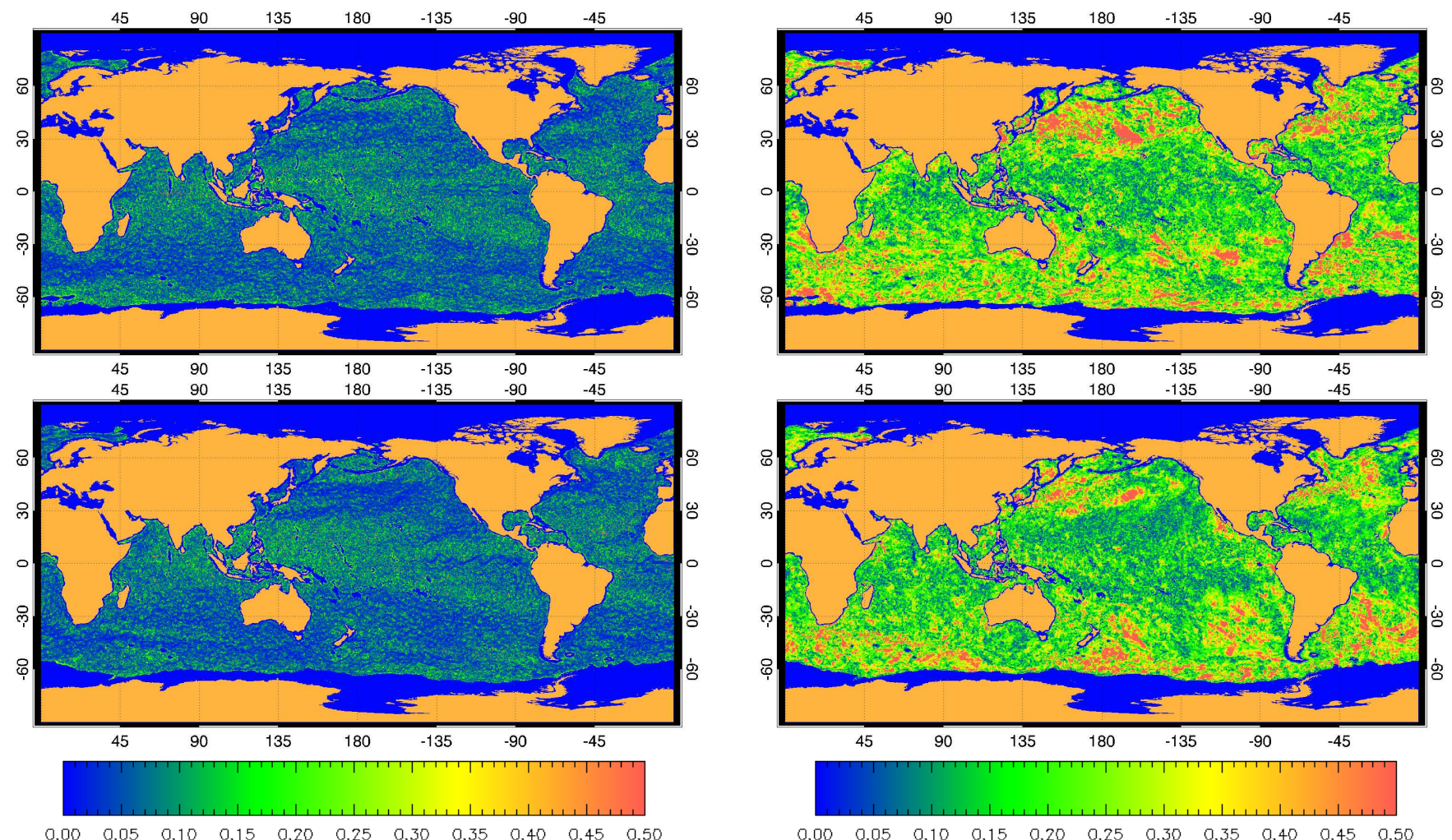

day $^{-1}$

${ }^{\circ} \mathrm{C} /$ day

Fig. 5. Time averages of absolute values of material derivatives of singularity exponent maps (left) and SST (right) for a period of three consecutive days. Results on the top row correspond to the period 1-3 January 2003; results on the bottom row are for 1-3 July 2003. The spatial averages of these quantities are as follows: Singularity exponents: 0.11 day $^{-1}$ (January) and 0.11 day $^{-1}$ (July); SST: $1.65^{\circ} \mathrm{C} / \mathrm{day}^{-1}$ (January) and $1.49^{\circ} \mathrm{C} /$ day (July).

we present the derivatives of both SST and singularity exponents.

As shown in the figures, both time derivatives are close to zero in the case of singularity exponents, although advective derivatives are significantly smaller. This means that the hypothesis that singularity exponents delineate streamlines is more consistent than the hypothesis of passive advection of singularity exponents. However, the partial time derivative of the singularity exponents, i.e., the term $\partial_{t} h$, is relatively small and so both types of derivative are not so different; hence, the hypothesis of passive advection of singularity exponents can be appropriate for short time periods. Comparing the results of the time derivatives of singularity exponents and those of SST is not straightforward, as they do not have the same units. Time derivatives of SST seem to be much less uniform than those of singularity exponents and significantly greater in value, but lacking of an appropriate conversion unit the used colorbars are conventional and so this conclusion is rather arbitrary. In fact, average advective derivatives of SST are of about $0.5^{\circ} \mathrm{C} /$ day, which do not seem very large. To help comparison, we have defined new quantities with the same dimensions for both variables and informative about the quality as fluid tracers of each variable. We thus define the advective divergence speed, $V_{A}$, and the material divergence speed, $V_{M}$, of a scalar $\theta$ as follows:

$$
V_{A}(\mathbf{x}, t) \equiv \frac{|A \theta(\mathbf{x}, t)|}{|\nabla \theta(\mathbf{x}, t)|} \quad, \quad V_{M}(\mathbf{x}, t) \equiv \frac{|D \theta(\mathbf{x}, t)|}{|\nabla \theta(\mathbf{x}, t)|}
$$

These quantities have units of speed, and we interpret them as the speeds at which the isolines of $\theta$ separate from the actual streamlines. This interpretation is supported by the implicit function identity $\partial_{t} \theta / \partial_{x} \theta=-\partial_{t} x$. A more precise argument in support of this interpretation is the following: the advective (vs. material) time derivative informs us about the rate of variation of the variable $\theta$ as we move along the streamline (vs. trajectory), but gives no idea about the distance that the water parcel has run to observe such an increment of the variable. On the other hand, the gradient of $\theta$ gives information about the spatial variability of $\theta$ going along the direction of fastest variation, which is always perpendicular to the isolines of $\theta$. Hence, the ratio of the time derivative by the gradient gives us the speed at which the streamline (vs. 

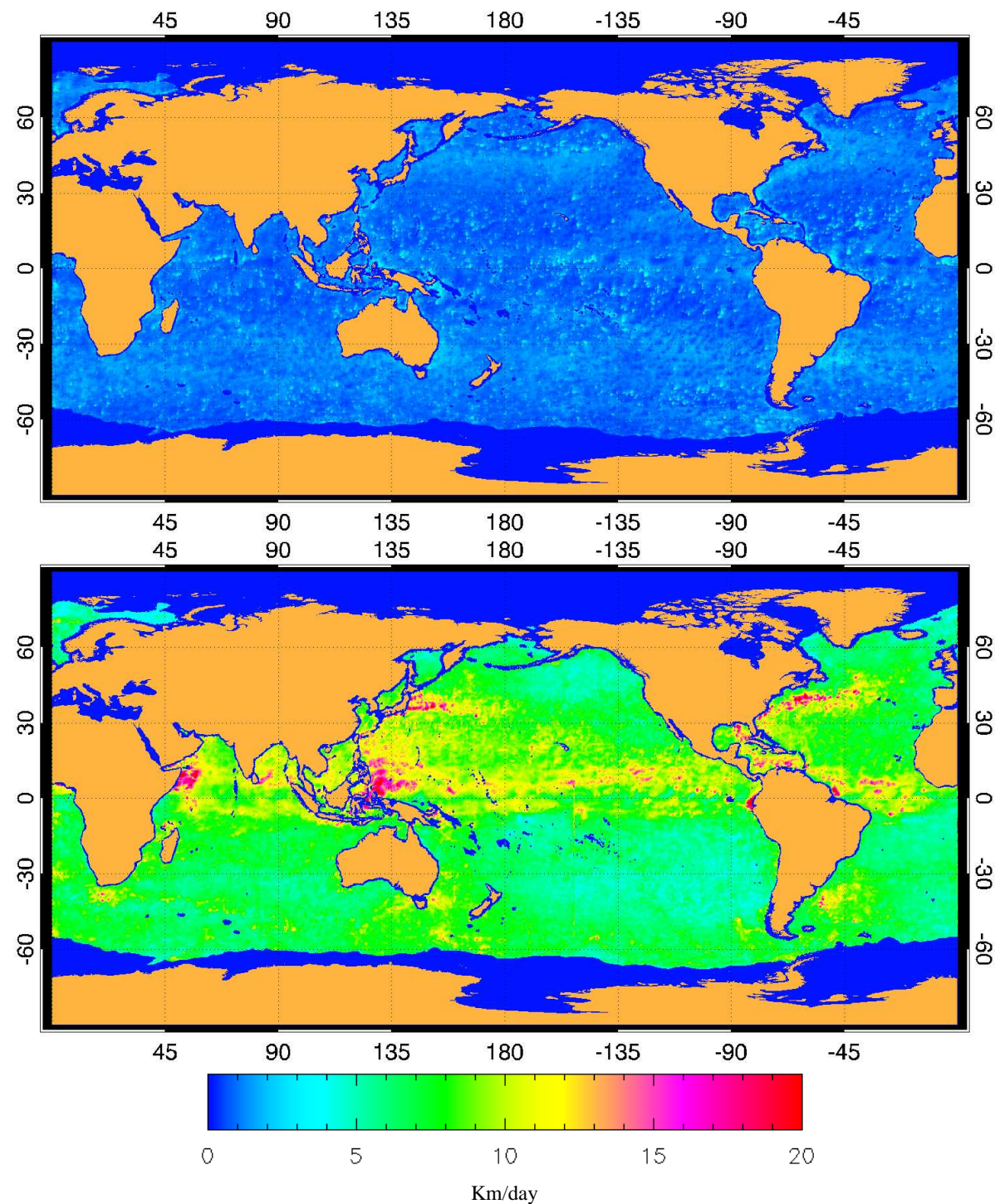

Fig. 6. Advective divergence speed maps obtained from singularity exponents (top) and from SST (bottom); results are for the time average over the days 1 to 3 July 2003. The spatially averaged advective divergence speeds are $1.03 \mathrm{Km} /$ day for singularity exponents and $7.59 \mathrm{Km}$ /day for SST.

trajectory) separates from a given spatial (vs. time-space) isoline of $\theta$. Reciprocally, we can interpret the divergence speed as the rate at which we separate from a streamline (vs. trajectory) if we follow the isoline of $\theta$ which passed by the same initial point.

Divergence speeds are robust measurements on the capabilities of a given scalar to trace streamlines or trajectories, and solve problems such as having different sampling grids for velocities and scalars. As only the integrated action of the velocity is taken into account in our estimates of time derivatives, the effect of spatially incoherent, noise-induced perturbations is greatly diminished. Alternative methods based on punctual measurements like measuring the angle between the gradient of the scalar and the velocity vector are rather ill-behaved. For instance, trying to estimate the angle between gradient and velocity could give a poor idea about the tracing capability of a scalar just because the gradient of the scalar at a given point is severely affected by noise or difficult to estimate due to intermittency, even if at that place velocity is small and tracing the streamline is still possible. Additionally, given a maximum acceptable spatial separation, dividing it by the divergence speed we obtain a time horizon for which our estimate of streamline can be considered good enough. Finally, tracing by the scalar is perfect if and only if the divergence speed is zero. The evaluation of divergence speeds on real, discretized data imply 

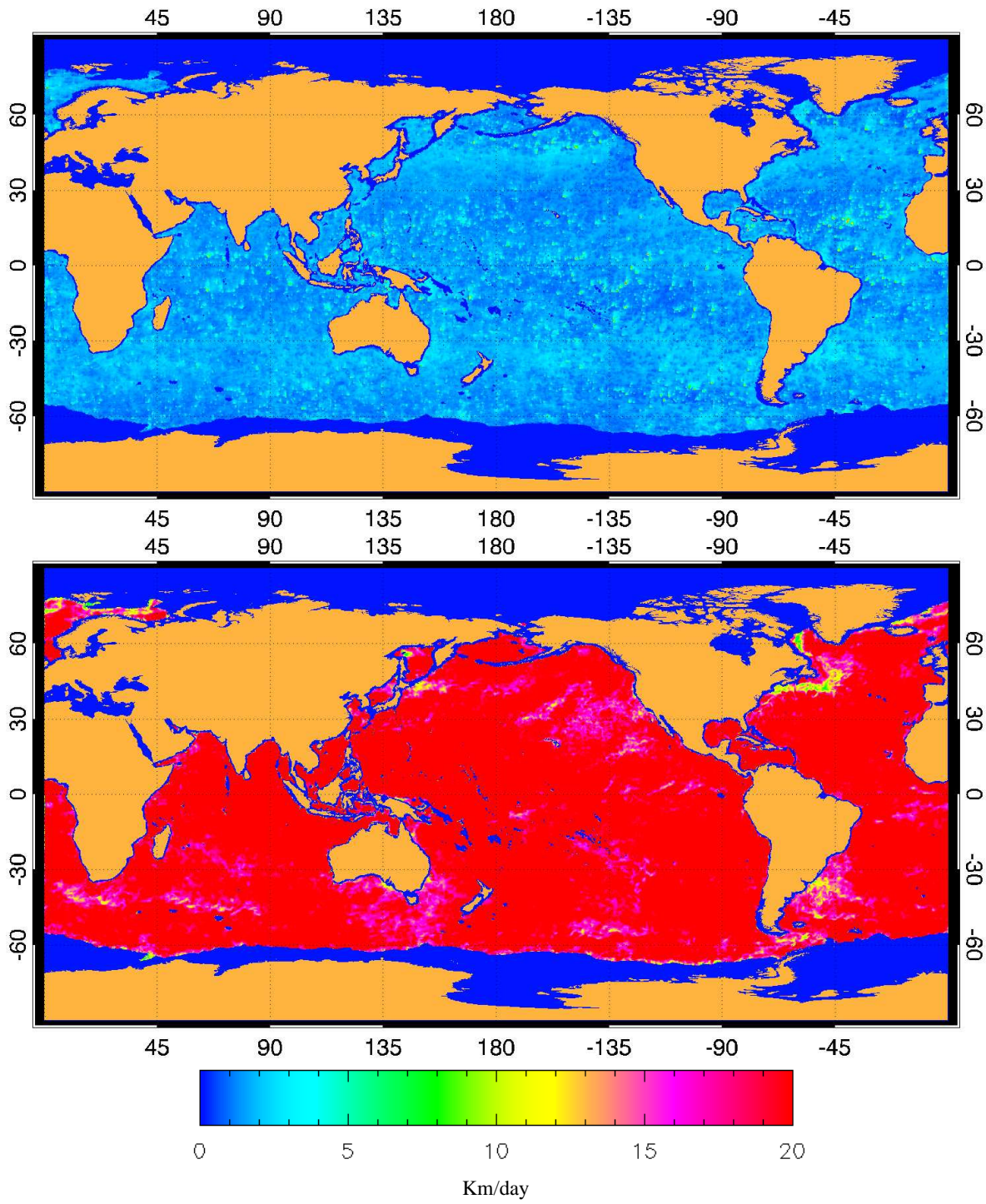

Fig. 7. Material divergence speed maps obtained from singularity exponents (top) and from SST (bottom); results are for the time average over the days 1 to 3 July 2003. The spatially averaged material divergence speeds are $1.61 \mathrm{Km} /$ day for singularity exponents and $23.57 \mathrm{Km} /$ day for SST.

nevertheless some processing, apart from the use of appropriate integration schemes for the velocity trajectories. Particularly, to avoid divergences due to cancellations in the gradient in Eq. (13), both the time derivative and the gradient are weighted with a fast-decreasing, almost scale-invariant kernel, namely $\left(1+|\mathbf{r}|^{2}\right)^{-1}$.

We show examples of divergence speeds in Figs. 6 and 7. Figures show that both advective and material divergence speeds of singularity exponents have small values, which are of the order $1-2 \mathrm{Km} /$ day on average, that is, around $1-2 \mathrm{~cm} / \mathrm{s}$. These values are similar to the uncertainty on the altimeterderived velocities, so that these divergence speeds have approximately the minimum possible value and are compat- ible with zero divergence speed. In addition, singularityderived divergence speeds are very uniformly distributed on the Globe, with more significant deviations on areas of larger mesoscale activity around the great boundary currents.

The situation is quite different for SST-derived divergence speeds. Advective divergence speeds are relative small on average (around $8 \mathrm{Km} /$ day) but significant, and less uniformly distributed that their singularity counterparts. Material divergence speeds, on the other hand, have rather large values, of order $30 \mathrm{Km} /$ day on average, with peaks up to $50 \mathrm{Km} /$ day (not shown in the figure, as the color scale is saturated to present singularity-derived and SSTderived maps with the same scale). The largest values of 


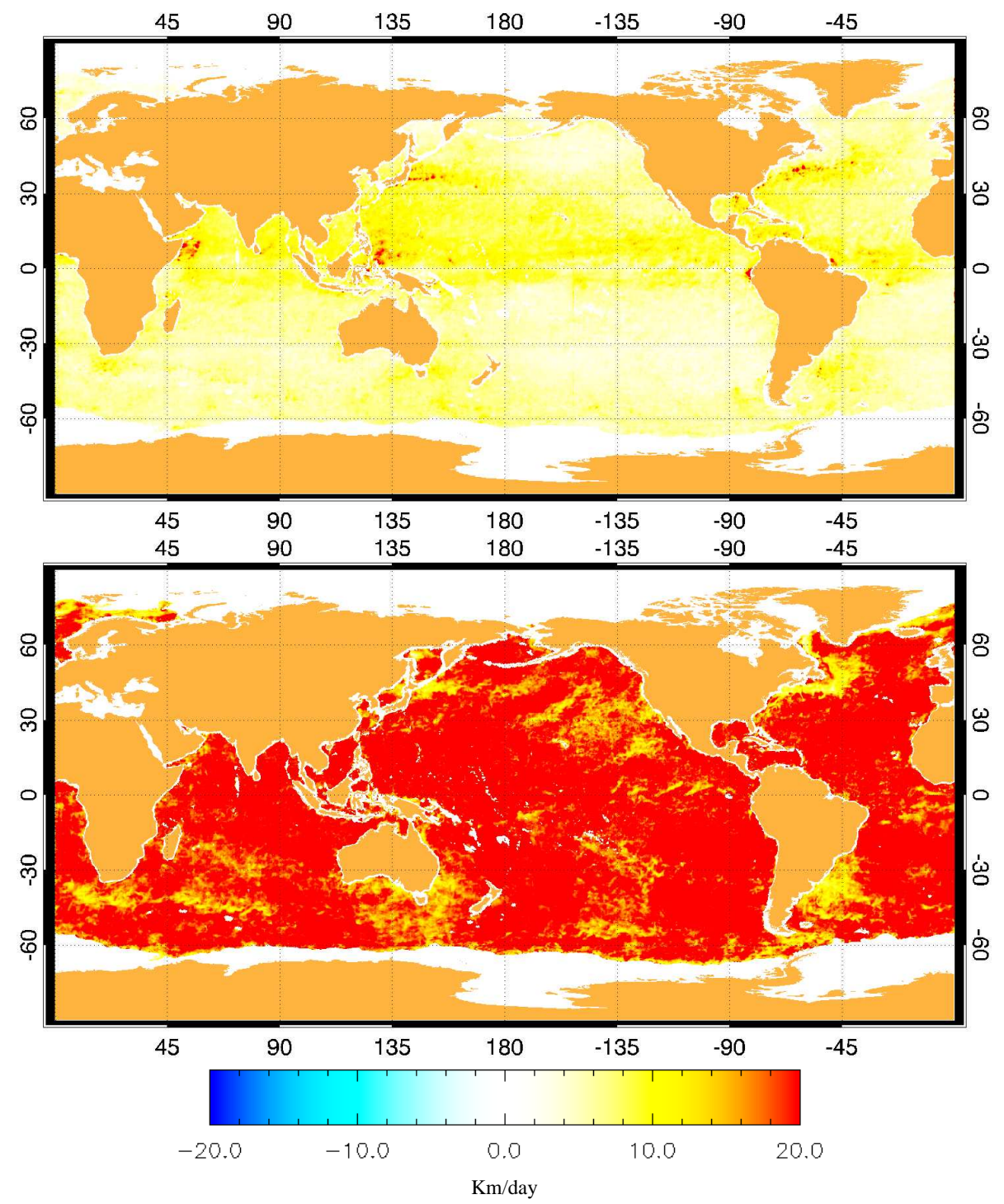

Fig. 8. Differences between the SST-derived and singularity-derived divergence speed maps. Top: Map of differences of the advective divergence speeds shown in Fig. 6. Bottom: Differences of the material divergence speeds shown in Fig. 7. Differences are positive at all points, meaning that SST-derived speeds are substantially greater at any place than their singularity-derived counterparts.

SST-derived material divergence speeds are associated to some frontal areas and possibly to the presence of active upwelling/downwelling, which indeed changes the thermal signature on the affected areas.

Although SST-derived divergence speeds are on average much greater than their singularity-derived counterparts, it could happen that at some particular places the opposite situation occurs, meaning that under special conditions SST is a better tracer than singularity exponents. In fact, this is never the case, as illustrated by Fig. 8: at the studied scale, divergence speeds for singularities are much smaller at any place, and also for the different times we have studied. Such a robust behavior reveals that singularity analysis is able to effi- ciently filter away a significant part of the dynamics of SST not related to advection, such as heat fluxes, diffusion, etc.

A different, altough less precise, measurement of the degree of closeness between the isolines of a given scalar and the altimetry streamlines is to measure their mutual angle. This quantity can be simply derived from the advective divergence speed, as:

$V_{A}(\mathbf{x}, t) \equiv \frac{|A \theta(\mathbf{x}, t)|}{|\nabla \theta(\mathbf{x}, t)|}=|\mathbf{v}(\mathbf{x}, t)||\cos \phi(\mathbf{x}, t)|$

where $\phi(\mathbf{x}, t)$ is the angle between the gradient of $\theta$ and the velocity field $\mathbf{v}$. Noticing that the gradient is perpendicular to the isolines of $\theta$, so at each point and time instant we 

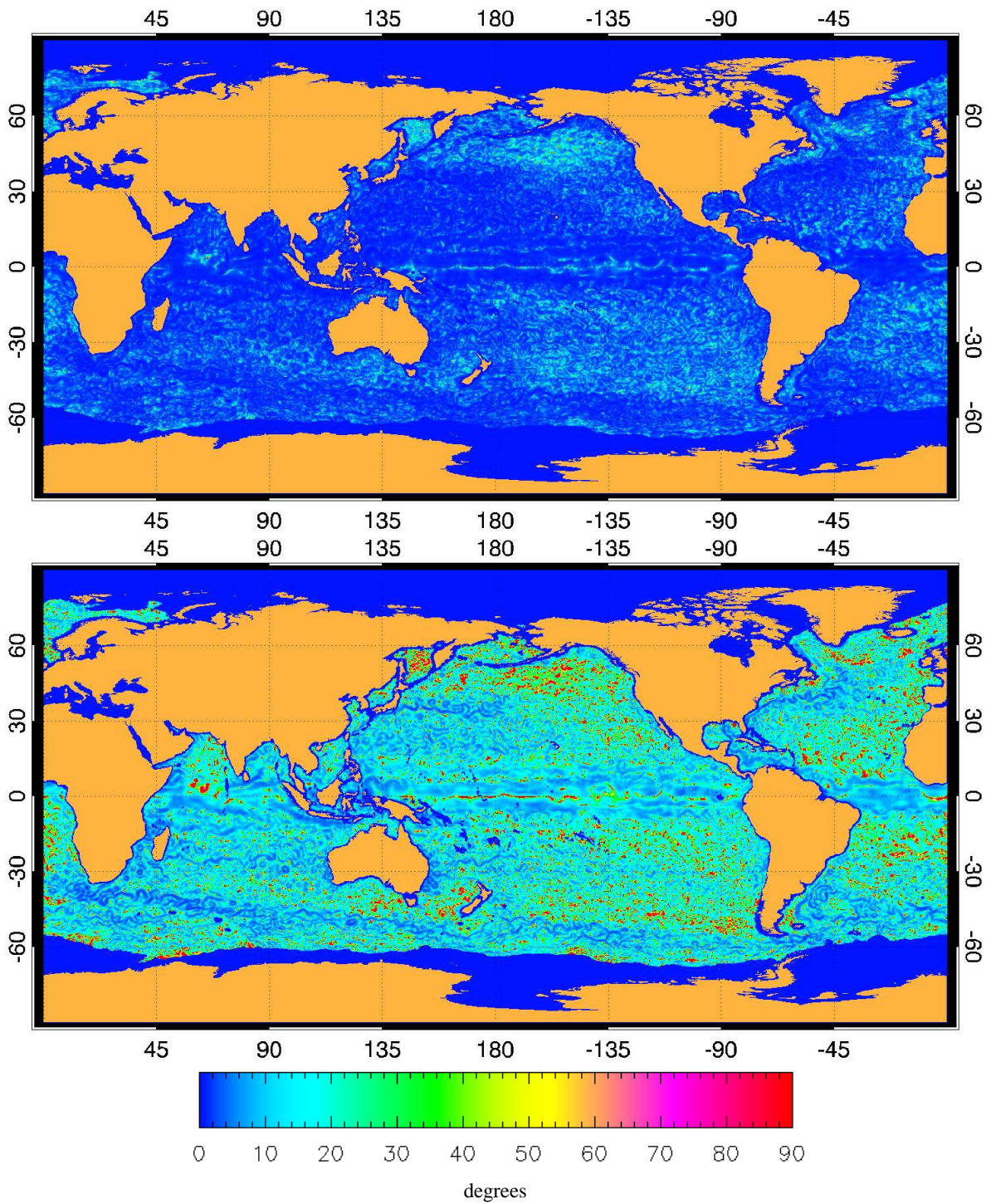

Fig. 9. Deviation angle between streamlines and scalar isolines. Top: Deviation angle averaged over the days 1 to 3 July 2003 for the singularity maps (spatial average: $3 \cdot 4^{\circ}$ ). Bottom: Deviation angle averaged over the days 1 to 3 July 2003 for SST (spatial average: $22.5^{\circ}$ ).

can compute the deviation angle between the directions of the isoline and that of the streamline applying the following expression.

$\phi(\mathbf{x}, t)=\sin ^{-1}\left(\frac{V_{A}(\mathbf{x}, t)}{|\mathbf{v}(\mathbf{x}, t)|}\right)$

This expression is more ill-behaved than the divergence speed, as it implies dividing the later by an extra term, which in turn is not necessarily defined over the same grid of the scalar $\theta$. Besides, small values of the velocity can give rise to large deviations in angle, even if the additive noise is rather small, due to the non-linear character of Eq. (15). Nevertheless results are quite convincing. In Fig. 9 we show an example of such deviation angles. As expected, the angle formed by the intersection of streamlines with isolines of singularities are considerably smaller than the angle of the intersection of streamlines with isotherms (spatial averages of $3.4^{\circ}$ and $22.5^{\circ}$, respectively). In addition, the angles formed by singularity isolines almost never exceed $25^{\circ}$ (probability less than $1 \%$ ), the outliers being attributed to structures missed by the singularity detection algorithm and coastal effects.

\section{Conclusions}

In this paper we have shown that singularity analysis applied to MW SST images can be used to uncover the circulation patterns in the global ocean. Singularity exponents are dimensionless quantities, and they are less affected by 
large-area effects like sun heating cycles, sunglint, etc. In addition, they have much richer spatial structure, with strong variations, what aids to give a precise location to mesoscale features like eddies and filaments. To demonstrate the ability of singularity analysis to reveal the actual surface streamlines we have compared them with a new branch of altimetry products (SURCOUF maps), which have been produced for a short period with optimal satellite coverage. SURCOUF maps are daily maps, what is essential to precisely estimate singularity advection as it has been shown that mesoscale oceanic features extracted by singularity analysis significantly evolve in a three-day period. In previous works altimeter-based evidence supporting that singularity lines trace streamlines had already been presented, but due to the limitations of the employed altimetry maps verification was limited to very active areas, and results were rather rough. On the contrary, in this work we have shown that there is a fine correspondence between SST-derived singularity isolines and SURCOUF surface streamlines. This work can hence be considered a cross-validation of both the singularity analysis method and SURCOUF products.

Results indicate that singularity exponents are appropriate to delineate instantaneous streamlines with an average uncertainty, measured in terms of the new concept of divergence speed, of about $1 \mathrm{Km} /$ day. This means that the rate at which we could expect to separate from the actual streamline if we follow a singularity isoline passing through the same initial point is of about $1 \mathrm{Km} /$ day on average. This value of divergence speed is about the smallest possible one, as it is of the order of altimeter accuracy. What is also important, the dispersion around this average value of divergence speed is quite narrow. Our results mean a significant improvement with respect to other techniques employed to extract dynamic information on SST such as MCC (Bowen et al., 2002) or Surface Quasi Geostrophy (Isern-Fontanet et al., 2006). However, singularity analysis does not provide access to the full velocity vector, just to its direction. The modulus and sense of this field can be guessed under the appropriate hypothesis (Turiel et al., 2005b) or integrating additional information (Isern-Fontanet et al., 2007). In this sense the combination with the information of the forthcoming generation of wide-swath altimeters (Chelton, 2001) will mitigate such a limitation providing an improvement of the results.

As MW SST images are now produced at a daily rate, the techniques described in this paper are relevant for many purposes. At a operational level it can eventually produce high-resolution operational instantaneous velocity fields. At a more fundamental level it enables a better use of satellite information to study many oceanographic processes. We can easily determine the position of different fronts associated to the Antarctic Circumpolar Current, to quantify the extent and propagation speed of Tropical Instability Waves or to study the filamentation of the great boundary currents and how they close the great subtropical gyres at the eastern boundaries. All those structures are strongly linked to large-extent phe- nomena which condition climate; the re-analysis of existing databases and the on-going produced maps will be useful to understand the short-term variability of oceanic part of the climate engine and to improve our knowledge in future years.

Acknowledgements. This is a contribution to the EU MERSEA project (AIP3-CT-2003-502885) and to the Spanish projects OCEANTECH (PIF 2006 project) and MIDAS-4 (ESP200506823-C05-1).

Edited by: B. Barnier

\section{References}

Arneodo, A., Argoul, F., Bacry, E., Elezgaray, J., and Muzy, J. F.: Ondelettes, multifractales et turbulence, Diderot Editeur, Paris, France, 1995.

Bowen, M., Emery, W., Wilkin, J., Tildesley, P., Barton, I., and Knewtson, R.: Extracting multiyear surface currents from sequential thermal imagery using the Maximum Cross-correlation Technique, J. Atmos. Oceanic Technol., 19, 1665-1676, 2002.

Chapron, B., Collard, F., and Ardhuin, F.: Direct measurement of ocean surface velocity from space: Interpretation and validation, J. Geophys. Res., 110, C0022, doi:doi:10.1029/2004JC0022, 2005.

Chelton, D. B.: Report of the High-Resolution Ocean Topography, Tech. rep., Science Working Group Meeting Report, http://www. coas.oregonstate.edu/research/po/research/hotswg/, 2001.

Crocker, R., Emery, W., Matthews, D., and Baldwin, D.: Computing Ocean Surface Currents from Infrared and Ocean Color Imagery, IEEE Trans. Geosci. Rem. Sens., 45, 435-447, 2007.

Daubechies, I.: Ten lectures on wavelets, CBMS-NSF Series in App. Math., Capital City Press, Montpelier, Vermont, 1992.

Frisch, U.: Turbulence, Cambridge Univ. Press, Cambridge MA, 1995.

Isern-Fontanet, J., Chapron, B., Lapeyre, G., and Klein, P.: Potential use of microwave sea surface temperatures for the estimation of ocean currents, Geophys. Res. Lett., 22, L24608, doi:10.1029/2006GL027801, 2006.

Isern-Fontanet, J., Turiel, A., Garcia-Ladona, E., and Font, J.: Microcanonical Multifractal Formalism: application to the estimation of ocean surface velocities, J. Geophys. Res., 112, C05024, doi:10.1029/2006JC003878, 2007.

Johannessen, J., Kudryavtsev, V., Akimov, D., Eldevik, T., Winther, N., Johannessen, O., and Chapron, B.: On Radar Imaging of Current Features; Part 2: Mesoscale Eddy and Current Front detection, J. Geophys. Res., 110, C07017, doi:10.1029/2004JC002802., 2005.

Kraichnan, R.: Small-scale structure of a scalar field convected by turbulence, Phys. Fluids, 11, 945-963, 1968.

Lagerloef, G. S. E., Mitchum, G., Lukas, R., and Niiler, P.: Tropical Pacific near surface currents estimated from altimeter, wind and drifter data, J. Geophys. Res., 104, 23313-23326, 1999.

Lapeyre, G., Hua, B., and Klein, P.: Dynamics of the orientation of active and passive scalars in two dimensional turbulence, Phys. Fluids, 13, 251-264, 2001.

Larnicol, G., Guinehut, S., Rio, M.-H., Drevillon, M., Faugere, Y., and Nicolas, G.: The global observed ocean products of the French Mercator project, in: Proceedings of the " 15 years 
of progress in Radar altimetry” ESA Symposium, ESA, Venice, 2006.

LeTraon, P. and Dibarboure, G.: An illustration of the unique contribution of the TOPEX/Poseidon - Jason-1 tandem mission to mesoscale variability studies, Marine Geodesy, 27, 3-13, 2004.

LeTraon, P., Nadal, F., and Ducet, N.: An improved mapping method of multisatellite altimeter data, J. Atmos. Oceanic Technol., 15, 522-534, 1998.

LeTraon, P., Faugère, Y., Hernandez, F., Dorandeuand, J., Mertz, F., and Ablain, M.: Can we merge GEOSAT Follow-On with TOPEX/POSEIDON and ERS-2 for an improved description of the ocean circulation?, J. Atmos. Oceanic Technol., 20, 889-895, 2003.

Mallat, S.: A Wavelet Tour of Signal Processing, Academic Press, 2nd Edition, 1999.

Nieves, V., Llebot, C., Turiel, A., Solé, J., García-Ladona, E., Estrada, M., and Blasco, D.: Common turbulent signature in sea surface temperature and chlorophyll maps, Geophys. Res. Let., 34, L23602, doi:10.1029/2007GL030823, 2007.

Pascual, A., Faugere, Y., Larnicol, G., and LeTraon, P.: Improved description of the ocean mesoscale variability by combining four satellite altimeters, Geophys. Res. Lett., 33, 611, doi:10.1029/2005GL024633, 2006.

Reynolds, R. and Smith, T.: Improved global sea surface temperature analyses using optimal interpolation, J. Climate, 7, 929-948, 1994.
Rio, M.-H., Schaeffer, P., et al.: The estimation of the ocean Mean Dynamic Topography through the combination of altimetric data, in-situ measurements and GRACE geoid: From global to regional studies, in: Proceedings of GOCINA, Luxembourg, 2005.

Turiel, A. and Parga, N.: The multi-fractal structure of contrast changes in natural images: from sharp edges to textures, Neural Computation, 12, 763-793, 2000.

Turiel, A., Grazzini, J., and Yahia, H.: Multiscale techniques for the detection of precipitation using thermal IR satellite images, IEEE Geosci. Rem. Sens. Let., 2, 447-450, doi:10.1109/LGRS.2005.852712, 2005a.

Turiel, A., Isern-Fontanet, J., García-Ladona, E., and Font, J.: Multifractal method for the instantaneous evaluation of the stream function in geophysical flows, Phys. Rev. Lett., 95, 104502, doi:10.1103/PhysRevLett.95.104502, 2005b.

Turiel, A., Solé, J., Nieves, V., Ballabrera-Poy, J., and GarcíaLadona, E.: Tracking oceanic currents by singularity analysis of Micro-Wave Sea Surface Temperature images, Rem. Sens. Environ., 112, 2246-2260, 2008a.

Turiel, A., Yahia, H., and Pérez-Vicente, C.: Microcanonical Multifractal Formalism: a geometrical approach to multifractal systems. Part I: Singularity analysis, J. Physics A, 41, 015501, doi: 10.1088/1751-8113/41/1/015501, 2008b.

Wunsch, C. and Stammer, D.: Satellite altimetry, the marine geoid, and the oceanic general circulation, Annu. Rev. Earth Planet. Sci., 26, 219-353, 1998 . 